\title{
Propensity-score matched comparison of partial versus radical nephrectomy for T1NOMO sarcomatoid renal cell carcinoma
}

\author{
Bing Ji ${ }^{1 \#}$, Dawei $\mathrm{Li}^{2 \#}$, Shuai $\mathrm{Fu}^{3 \#}$, Zhao Zhang ${ }^{2}$, Tong Yang ${ }^{2}$, Yaohai $\mathrm{Wu}^{2}$, You Zuo ${ }^{2}$, Zhonghua $\mathrm{Xu}^{2}$, \\ Nengwang $\mathrm{Yu}^{2}$ \\ ${ }^{1}$ School of Control Science and Engineering, Shandong University, Jinan 250061, China; ${ }^{2}$ Department of Urology, Qilu Hospital of Shandong \\ University, Jinan 250012, China; '3Shandong Cancer Hospital and Institute, Shandong Academy of Medical Sciences, Jinan 250000, China \\ Contributions: (I) Conception and design: N Yu; (II) Data analysis and interpretation: B Ji, D Li, S Fu; (III) Collection and assembly of data: Z Zhang, \\ T Yang, Y Wu, Y Zuo, Z Xu; (IV) Manuscript writing: All authors; (V) Final approval of manuscript: All authors. \\ "These authors contributed equally to this work. \\ Correspondence to: Nengwang Yu. Department of Urology, Qilu Hospital of Shandong University, 107 West Wenhua Road, Jinan 250012, China. \\ Email: yunengwang@hotmail.com.
}

Background: Sarcomatoid renal cell carcinoma is a rare variant of renal cell carcinoma associated with poor clinical outcomes. Currently no reliable evidence indicates whether partial nephrectomy can be an effective treatment for patients with T1N0M0 sarcomatoid renal cell carcinoma. The present study was conducted to compare the overall and cancer-specific survival with partial and radical nephrectomy for such cases.

Methods: Data of sarcomatoid renal cell carcinoma were retrieved from Surveillance, Epidemiology, and End Results database. Factors independently associated with the overall or cancer-specific survival were identified by Cox regression analysis. The overall and cancer-specific survival of propensity-score matched T1N0M0 sarcomatoid renal cell carcinoma patients treated by partial and radical nephrectomy were estimated by the Kaplan-Meier method.

Results: The records of 452 T1N0M0 sarcomatoid renal cell carcinoma cases were retrieved. One hundred and fifty-five of these patients underwent partial nephrectomy, while the remaining 297 underwent radical nephrectomy. The median follow-up period in partial-treated group was 38 months (ranging from 2 to 132 months), while that in RN-treated was 39 months (ranging from 0 to 150 months). Age, sex, marital status and tumor size were independent risk factors for the overall and cancer-specific survival. There were no significant differences among age, sex, marital status and tumor size in the PN- and RN-treated T1N0M0 cases according to propensity-score matching. The estimated median overall survival of partial-treated group was 132 months, while that for $\mathrm{RN}$-treated cases was 100 months $(\mathrm{P}=0.11)$. The median cancer-specific survival was not reached in both groups $(\mathrm{P}=0.092)$.

Conclusions: The overall and cancer-specific survival of T1N0M0 patients treated by partial nephrectomy was not inferior to that of patients treated by radical nephrectomy.

Keywords: Sarcomatoid renal cell carcinoma; nephrectomy; survival

Submitted Nov 14, 2019. Accepted for publication Feb 14, 2020.

doi: $10.21037 /$ tau.2020.02.19

View this article at: http://dx.doi.org/10.21037/tau.2020.02.19

\section{Introduction}

The annual incidence of renal cell carcinoma (RCC), the form of renal tumor most frequently suffered by adults, is rising (1). Although clear cell, papillary and chromophobe
RCC account for most diagnoses, other histological subtypes with type-specific clinicopathological characteristics and prognoses may also present. Partial nephrectomy $(\mathrm{PN})$ is the preferred treatment recommended by current guidelines 
for patients with T1N0M0 RCC, and histological subtypes are not taken into consideration in this recommendation (2). Sarcomatoid renal cell carcinoma (SRCC), a rarely encountered variant of RCC, comprises about $5 \%$ of RCC cases. SRCC arises from any subtype of epithelial RCC, such as clear cell, papillary, or chromophobe, and presents as a deeply dedifferentiated tumor (3). SRCC is associated with poor clinical outcomes and advanced clinicopathological features (4). Currently there is no reliable evidence that supports the recommendation that $\mathrm{PN}$ can be an effective option for T1N0M0 SRCC. In the present study, cases of T1N0M0 SRCC were retrieved from the National Cancer Institute's Surveillance, Epidemiology, and End Results (SEER) program to inform comparisons between the differences in the survival of patients treated by either PN or radical nephrectomy (RN) after adjusting for other variables influencing survival.

\section{Methods}

\section{Data source and study population}

A cohort of SRCC patients diagnosed between 2004 and 2015 was established based on data obtained from SEER, which brings together high-quality data from 18 cancer registries, accounting for about $27.8 \%$ of Americans according to the United States' 2010 census (5). SRCC includes the union of histologic type ICD-O-3 8318 (RCC, sarcomatoid) and conventional RCC with Kidney Parenchyma CS Site-Specific Factor 4 code 010, while conventional RCC includes the union of histologic type ICD-O-3 8310 [clear cell adenocarcinoma, not specified (NOS)], 8312 (RCC), 8260 (papillary adenocarcinoma, NOS), 8317 (RCC, chromophobe type) and 8255 (adenocarcinoma with mixed subtypes). RCC, its size smaller than $7 \mathrm{~cm}$ and confined to the kidney was defined as stage $\mathrm{T} 1(\leq 4 \mathrm{~cm}$ as $\mathrm{T} 1 \mathrm{a}, 4-7 \mathrm{~cm}$ as $\mathrm{T} 1 \mathrm{~b})$ according to American Joint Committee on Cancer (AJCC) TNM staging system. Only "microscopically confirmed" RCC patients were included in our study. Cases without followup time or vital status were excluded. Ultimately, only patients with T1N0M0 SRCC were included.

\section{Patient variables}

Patient data were extracted from SEER database fields including "age", "race", "gender", "marital status at diagnosis", "histologic type ICD-O-3", "grade", "laterality", "derived AJCC stage group, 6th ed. (2004+)", "derived AJCC T, 6th ed. (2004+)", "derived AJCC N, 6th ed. (2004+)", "derived AJCC M, 6th ed. (2004+)", "RX SummSurg Prim Site (1998+)", "CS tumor size (2004+)", "CS site-specific factor 4 (2004+)", "SEER cause-specific death classification", "survival months" and "vital status recode (study cutoff used)."

\section{Propensity-score matching}

Multivariable Cox regression analysis was used to identify variables that significantly impact the overall survival (OS) or cancer-specific survival (CSS) among patients with T1N0M0 SRCC. The OS and CSS of T1N0M0 SRCC patients treated by either PN (derived from using RX Summ-Surg Prim Site code 30) or RN (derived from using RX Summ-Surg Prim Site code 40, 50, 80) were compared after propensity score matching (PSM) of patient cohorts to reduce potential confounding effects and the treatment selection bias. PSM was conducted by $\mathrm{R}$ version 3.5.3 and the MatchIt package (6). Propensity matching included variables significantly related to OS and CSS based on the results of multivariable Cox regression analysis. Nearestneighbor matching with a 1:1 ratio and a caliper distance of 0.2 was used.

\section{Statistical analysis}

The clinicopathological characteristics of the two propensity-score-matched groups of T1N0M0 SRCC patients treated by $\mathrm{PN}$ or RN were compared. The values of unordered categorical variables were compared by chi-square tests. Normality of the quantitative variables was conducted by Kolmogorov-Smirnov and ShapiroWilk normality tests. The values of unordered categorical variables were compared by chi-square tests. The unpaired Student's $t$-test was employed to compare the means of two continuous variables with a normal distribution. The Mann-Whitney $U$ test was used to compare continuous variables that did not have a normal distribution. The Kaplan-Meier estimator was utilized to estimate the cumulative survival, and the log-rank test was used to draw comparisons. The statistical analysis was performed with SPSS 25.0 for Windows (IBM Corp., Armonk, NY, USA). All tests were two-tailed, and significance was indicated when $\mathrm{P}<0.05$. 
Table 1 Patients' clinical and pathological characteristics at baseline

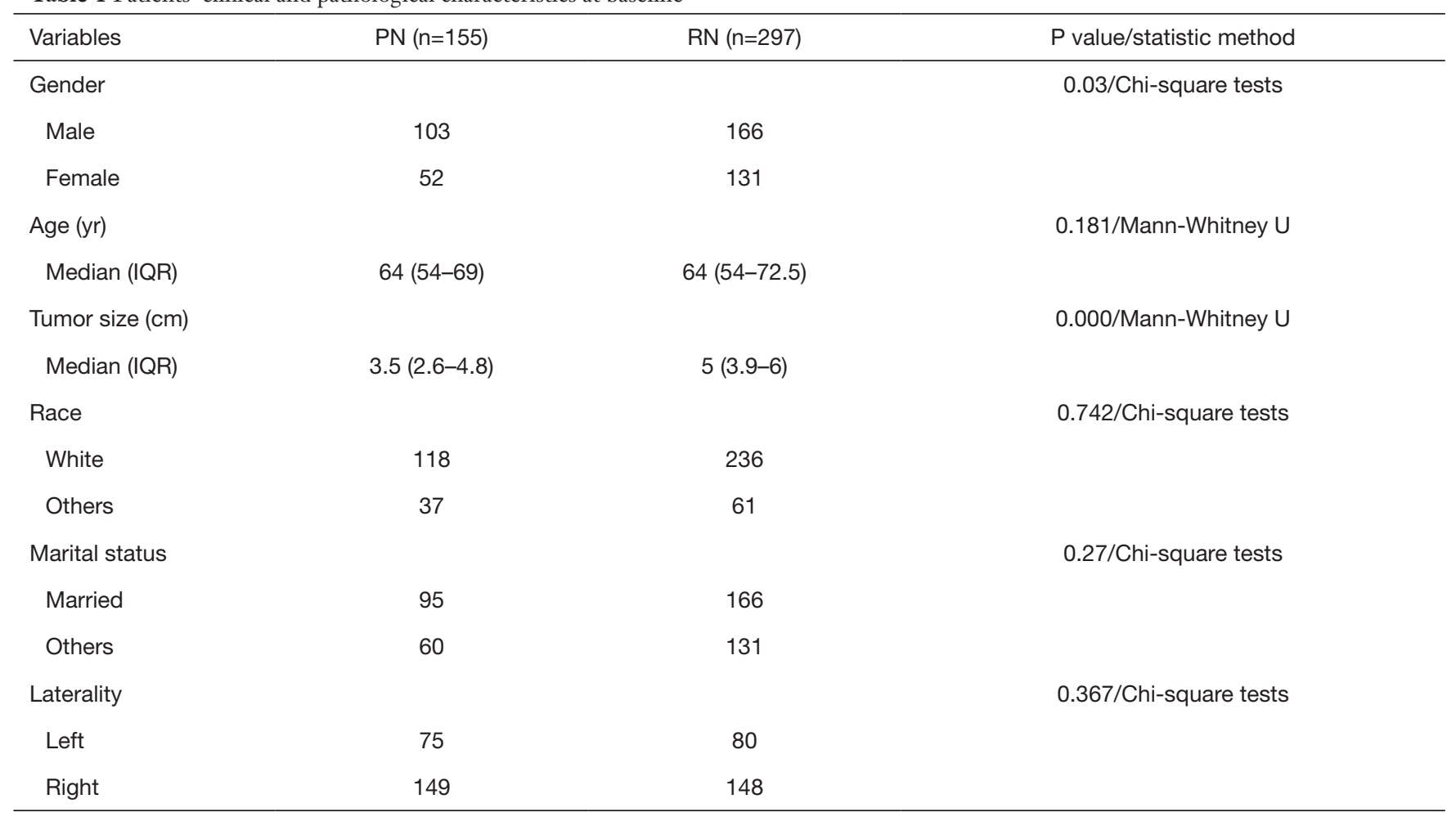

$\mathrm{Cl}$, confidence interval; PN, partial nephrectomy; RN, radical nephrectomy; IQR, interquartile range. Kolmogorov-Smirnov and ShapiroWilk normality tests were conducted and it was found that the data of tumor size and age was non-normally distributed.

\section{Results}

\section{Data}

A total of 3,655 SRCC cases of SRCC were identified on the SEER database, of which 452 were stage T1N0M0 (160 cases of T1aN0M0 and 292 cases of T1bN0M0). The median follow-up period in PN-treated T1N0M0 SRCC was 38 months (ranging from 2 to 132 months), while that in $\mathrm{RN}$-treated cases was 39 months (ranging from 0 to 150 months). Patients' clinical and pathological characteristics at baseline were shown in Table 1 .

\section{Outcomes of T1NOMO SRCC patients treated by PN or RN}

Age, sex, marital status and tumor size were shown to be independent factors associated with OS using multivariate Cox analysis, while tumor size was independently associated with CSS (as shown in Table 2). No significant differences were observed in age, sex, marital status, or tumor size between the PN- and RN-treated cases after PSM (as shown in Table 3).
As shown in Figure 1, there were no significant differences in the 5-year OS or CSS of T1NOM0 SRCC patients treated by PN or RN after PSM. The estimated median OS of PNtreated T1N0M0 SRCC was 132 months, while that of RNtreated cases was 100 months $(\mathrm{P}=0.11)$. The median CSS was not reached in both groups $(\mathrm{P}=0.092)$. The 5 -year OS was $85.0 \%$ in the $\mathrm{PN}$ group and $80.9 \%$ in the $\mathrm{RN}$ group, 5 -year CSS was $91.8 \%$ in the PN group and $87.2 \%$ in the $\mathrm{RN}$ group, respectively. We stratified T1 patients into T1a and T1b, and performed the further analysis. The survival benefit was found in PN-treated T1aN0M0 SRCC, but not in T1bN0M0 SRCC, compared to their RN-treated counterpart.

\section{Subgroup analysis}

We compared OS of PN- and RN-treated SRCC patients in T1aN0M0 and T1bN0M0 subgroups and in subgroups according to age ( $>60$ or $\leq 60$ years old), after balancing other variables impacting survival. A survival benefit was found in patients treated by $\mathrm{PN}$ in the T1aN0M0 subgroup, while 
Table 2 Univariate and Multivariate Cox regression of overall survival and cancer-specific survival in T1N0M0 SRCC patients

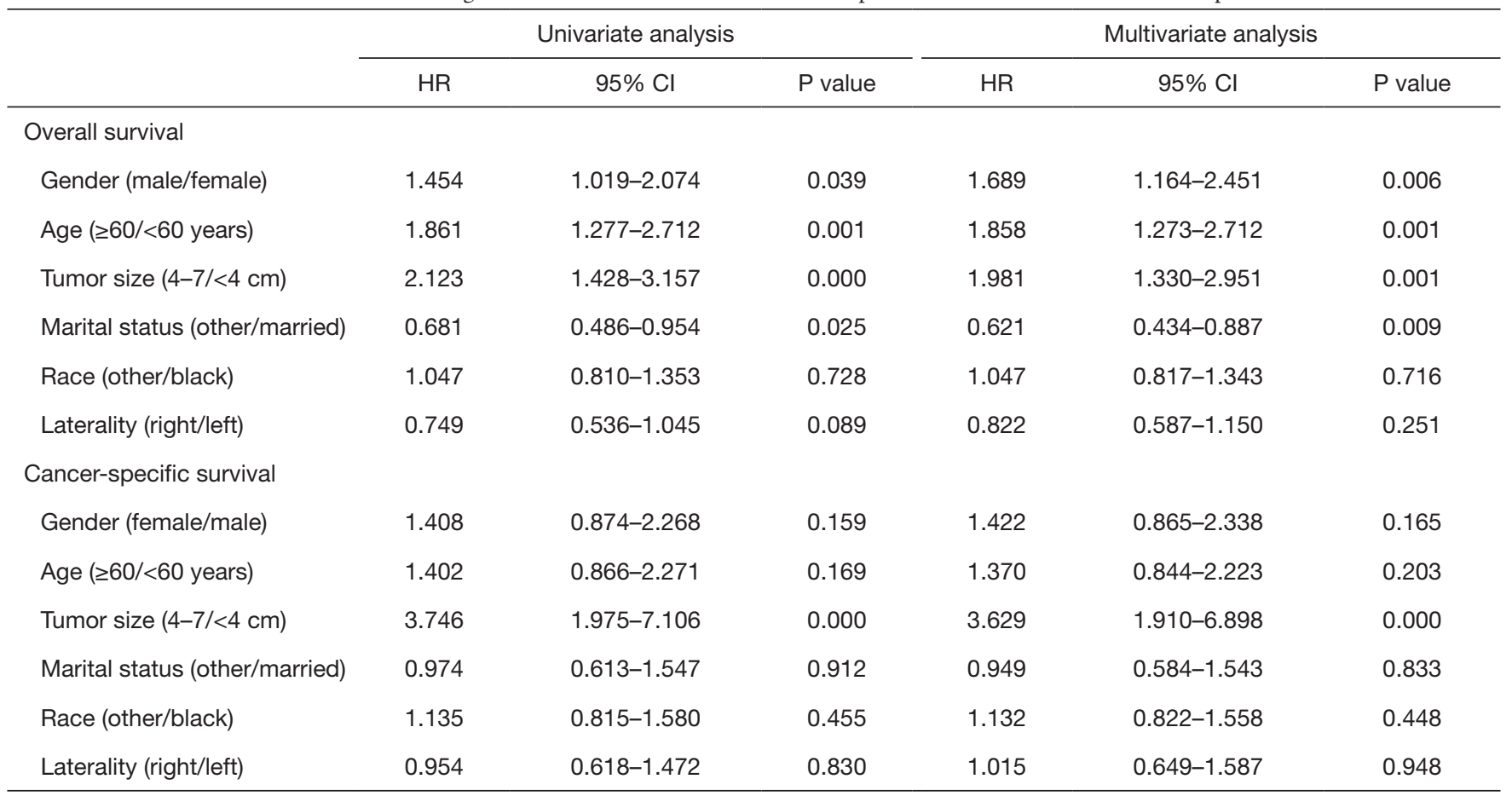

OS, overall survival; CSS, cancer-specific survival; $\mathrm{Cl}$, confidence interval; $\mathrm{HR}$, hazard ratio.

Table 3 Clinical and pathological characteristics of propensitymatched T1N0M0 SRCC patients treated by partial and radical nephrectomy

\begin{tabular}{lccc}
\hline Variables & $\begin{array}{c}\mathrm{PN} \\
(\mathrm{n}=155)\end{array}$ & $\begin{array}{c}\mathrm{RN} \\
(\mathrm{n}=155)\end{array}$ & $\begin{array}{c}\mathrm{P} \text { value/statistic } \\
\text { method }\end{array}$ \\
\hline Gender & & & $0.904 /$ Chi-square tests \\
Male & 103 & 102 & \\
Female & 52 & 53 & $0.94 /$ t-test \\
Age (years) & & & $0.341 / t$-test \\
Mean \pm SD & $61.5 \pm 11.3$ & $61.6 \pm 12.6$ & \\
Tumor size $(\mathrm{cm})$ & & & \\
Mean \pm SD & $3.71 \pm 1.43$ & $3.86 \pm 1.37$ & \\
Marital status & & & \\
Married & 95 & 87 & \\
Others & 60 & 68 &
\end{tabular}

$\mathrm{Cl}$, confidence interval; $\mathrm{PN}$, partial nephrectomy; RN, radical nephrectomy. Kolmogorov-Smirnov and Shapiro-Wilk normality tests were conducted and it was found that the data of tumor size and age was normally distributed. there was no significant difference in OS in T1bN0M0 SRCC or T1N0M0 SRCC between patients older or younger than 60 years treated by $\mathrm{PN}$ or RN (Figure 2).

\section{Outcomes of T1NOMO non-sarcomatoid RCC patients treated by PN or RN}

To compare with the SRCC cohort, we retrieved 25,886 cases of PN-treated and 19,332 cases of RNtreated T1aN0M0, while 5,541 cases of PN-treated and 17,871 cases of T1bN0M0 non-sarcomatoid RCC from contemporaneous SEER database. After adjusting other variables influencing the survival in $\mathrm{PN}$ - and $\mathrm{RN}$-treated groups, we found the survival advantage conferred by $\mathrm{PN}$ in both T1a and T1b subgroup. For T1aNOM0 and T1bN0M0 non-sarcomatoid RCC patients, the 5 -year OS was $91.2 \%$ and $86.7 \%$ in the PN group, while $84.7 \%$ and $85 \%$ in the RN group; the 5-year CSS was $99 \%$ and $96.7 \%$ in the $\mathrm{PN}$ group, while $97.5 \%$ and $96.3 \%$ in the $\mathrm{RN}$ group, respectively. When comparing the difference of OS or CSS between each given $\mathrm{PN}$-treated and $\mathrm{RN}$-treated groups, $\mathrm{P}$ value was all smaller than 0.05 (as shown in Figure 3). 
A

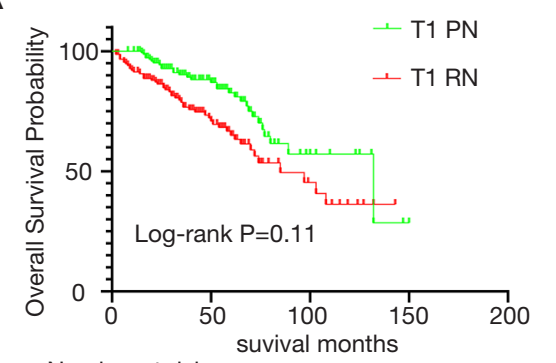

Number at risk

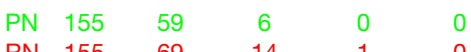

C

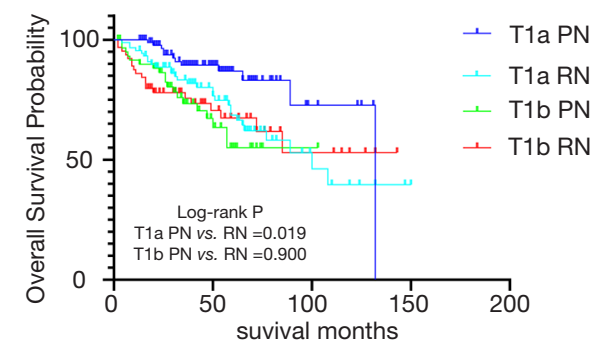

Number at risk

T1aPN $94 \quad 41 \quad 5 \quad 000$

T1bRN $89 \quad 45$

T1bPN $61 \quad 18$

T1bRN $66 \quad 24$

$\begin{array}{lll}5 & 0 & 0 \\ 8 & 1 & 0 \\ 1 & 0 & 0 \\ 6 & 0 & 0\end{array}$

B

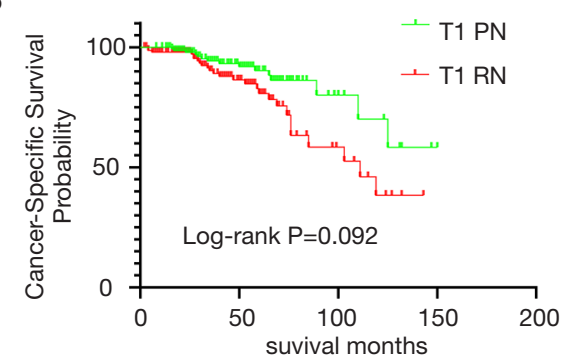

Number at risk

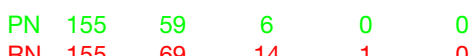

D

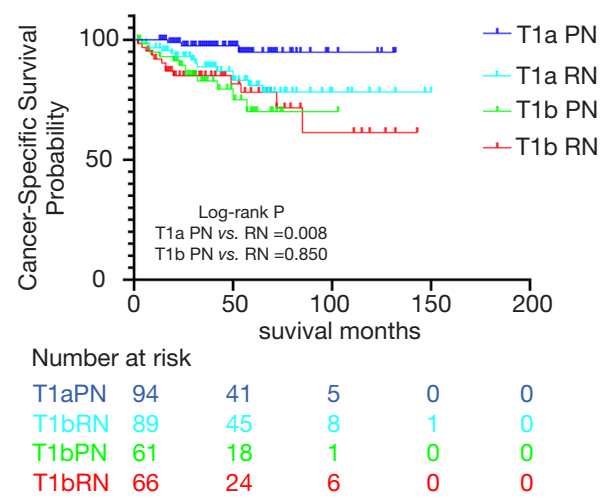

Figure 1 Kaplan-Meier overall survival (panel A for T1, panel C for T1a and T1b) and cancer-specific survival (panel B for T1, panel D for T1a and T1b) of T1N0M0 SRCC patients treated by partial and radical nephrectomy. PN, partial nephrectomy; RN, radical nephrectomy.

median os (months)
PN group (cases)
$132(94)$
NA(61)
NA(61)
$89(94)$

RN group(cases)

100(89)
$N A(66)$
$N A(63)$
$85(92)$

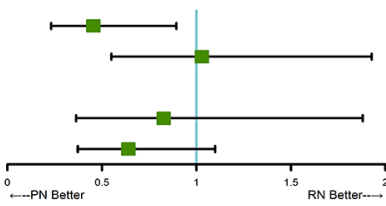

B

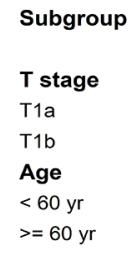

median OS (months) PN group (cases)

\section{2(94)}

NA(61)

$\mathrm{NA}(61)$

89(94)
RN group(cases)

100(89)

NA(66)

NA(63)

85(92)
Univariate HR (95\% CI)

$0.455(0.231-0.894)$

$1.029(0.55-1.927)$

$0.827(0.364-1.88)$

$0.64(0.372-1.099)$

Figure 2 Comparison of median overall survival of propensity score-matched different subgroups of sarcomatoid renal cell carcinoma patients treated by partial nephrectomy or radical nephrectomy. PN, partial nephrectomy; RN, radical nephrectomy; OS, overall survival; CI, confidence interval; HR, hazard ratio. 
A
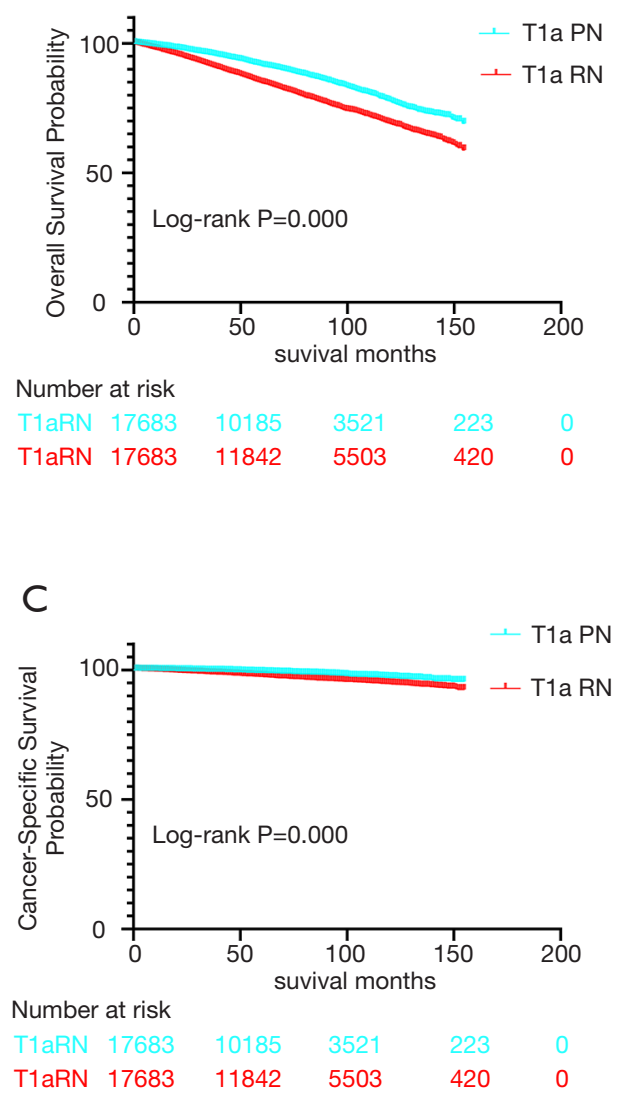

B

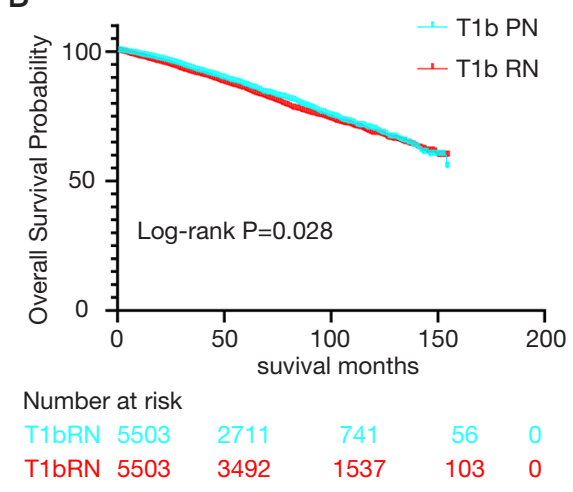

D

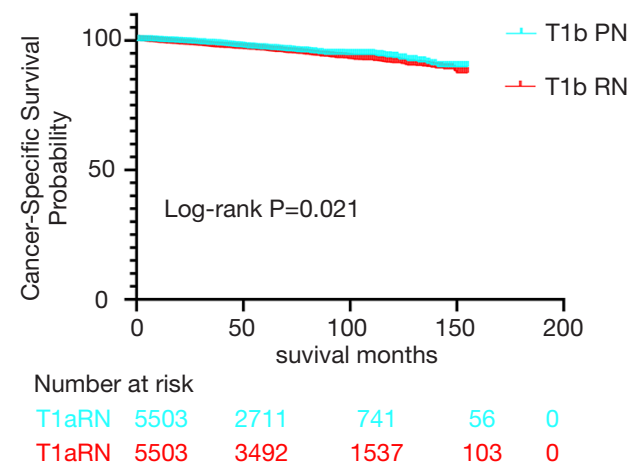

Figure 3 Kaplan-Meier overall survival (panel A for T1a, panel B for T1b) and cancer-specific survival (panel C for T1a, panel D for T1b) of T1N0M0 non-sarcomatoid RCC patients treated by partial and radical nephrectomy. PN, partial nephrectomy; RN, radical nephrectomy.

\section{Discussion}

Currently, PN is the preferred surgical treatment for T1N0M0 RCC in clinical practice, although the only existing randomized controlled trial (RCT), EORTC 30904, did not find that T1N0M0 RCC patients with a normal contralateral kidney treated by $\mathrm{PN}$ had superior OS compared to those treated by RN (7). Because patients with SRCC have the worst prognosis of all renal tumor patients (8), SRCC should be managed differently to conventional RCC. Empirically, PN is not recommended for patients with SRCC (3). Ideally, RCTs to compare PN and RN for T1N0M0 SRCC should be conducted. However, it is difficult to conduct such RCTs since there is currently no reliable preoperative method to identify SRCC (9). In clinical practice, few PNs are performed at a single center because the presence of large and bulky tumors can make the surgery impossible. For example, only 2 out of a series of 77 cases of localized SRCC at MD Anderson Cancer Center underwent PN (10). Therefore, it is difficult to systematically assess the outcomes of T1N0M0 SRCC treated by PN.

In this population-based study, 155 T1N0M0 SRCC patients who underwent PN were retrieved from the SEER database, forming the largest cohort of such cases reported to date. After adjusting for preoperative features associated with survival, the OS and CSS of the T1N0M0 patients treated by $\mathrm{PN}$ were not inferior to those of RN-treated patients. The survival benefit treated by $\mathrm{PN}$ was not found in T1N0M0 SRCC patients, while found in T1N0M0 non-sarcomatoid RCC in the present study. One possible cause for this result may be the differential prognostic role of histological feature. Although we found a survival benefit treated by PN in T1aN0M0 SRCC patients, it is still not clear whether this was result from the selection bias between $\mathrm{PN}$ - and $\mathrm{RN}$-treated groups or PN really providing superior OS in these patients. Several other retrospective analyses had demonstrated improvements in OS treated with PN in T1 RCC patients (11). However, 
Brian S el al. conducted a SEER-Medicare matched cohort study, which compared RCC surgical groups with separate controls, and found that the survival advantage conferred by $\mathrm{PN}$ was likely due to the selection bias (12). No survival difference existed between PN- and RN-treated T1N0M0 SRCC patients younger or older than 60 years, which is consistent with previous studies. Subgroup analysis of OS in EORTC 30904 trial of PN Versus RN also showed no difference in older or younger patients (13). An et al. also found OS and CSS were equivalent between PN- and RNtreated T1-T2 renal masses patients older than 65 years (14).

There are several limitations of the present study. First, the bias that impacted the survival of patients treated with $\mathrm{PN}$ and RN may exist, and might have not been completely removed using PSM, partly because of the limitations of SEER data. SEER data may be influenced by the variables, which are not recorded, not fully reported, incomplete, lacking uniformity in coding and reporting of data, or the movement of patients into or from the areas covered by the SEER program. Second, lacking information about the percentage of sarcomatoid component in each SRCC patient and the involvement of pathologists with different expertise limit the quality of pathology diagnosis. Previous studies have demonstrated that a greater percentage of sarcomatoid was associated with a worse outcome (15). Third, the difference between tumor mass complexity and surgical approaches or techniques may also impact the survival. However we had not balanced them between $\mathrm{PN}$ - and RN-treated groups, due to lacking information of nephrometry scores of renal mass (like RENAL or PADUA score), and the type of surgical approaches and techniques in SEER database. Fourth, based on the experience of Capitanio et al. (16), EAU guidelines recommend offering an extended lymph node dissection (eLND) to patients with adverse clinical features. There were only 9 patients in the $297 \mathrm{RN}$-treated, while no one in the $155 \mathrm{PN}$ treated SRCC patients who accepted eLND, if we regarded a cutoff of 4 or more regional lymph nodes removed as eLND. In this context, it is hard to analyse the impact of eLND on the present study. Sixth, in the cohort of SRCC patients retrieved for our study, only the sixth edition of AJCC TNM classifications could be retrieved, while several modifications have been made to the $\mathrm{T}$ and $\mathrm{N}$ stages in the current (eighth) edition. However, given that we only investigated patients with T1N0M0 RCC in the present study, it should not be significantly impacted by TNM stage modifications in the sixth, seventh, and eighth editions.

The present study was a retrospective analysis based on one public database, thus it just obtained results with the low-level evidence. The further investigation considering more databases data and multicenter prospective studies, is needed to verify the results of present study. If SRCC can be identified preoperatively in biopsy tissue, or with molecular biomarkers or imaging, then RCTs to compare the outcomes of PN versus RN for T1N0M0 SRCC may provide more definitive results. In the real world, where there is currently neither a reliable method for detecting SRCC preoperatively nor a large number of T1N0M0 SRCC cases treated with PN in single centers, results of our population-based study may supply the only evidence available for informed clinical decision making. For example, if SRCC is identified postoperatively in T1N0M0 tumors treated with $\mathrm{PN}$, some clinicians may wonder whether salvage radical surgery should be performed because of the infiltrative nature of SRCC. Based on the results of our present study, salvage radical surgery may not be necessary for patients treated with PN for a T1N0M0 renal mass that is confirmed postoperatively as SRCC.

\section{Conclusions}

Our study found that the OS and CSS of T1N0M0 patients treated by $\mathrm{PN}$ were not inferior to those of patients treated by $\mathrm{RN}$.

\section{Acknowledgments}

We thank AME Editing Service for editing this manuscript. Funding: This study was supported by financial grants from the National Natural Science Foundation of China (grant no. 61673246, 81902355), Natural Science Foundation of Shandong Province (grant no. ZR2018MH024) and the Focused Research and Development Program of Shandong Province (grant no. 2017GSF18130).

\section{Footnote}

Conflicts of Interest: All authors have completed the ICMJE uniform disclosure form (available at http://dx.doi. org/10.21037/tau.2020.02.19). The authors have no conflicts of interest to declare.

Ethical Statement: The authors are accountable for all aspects of the work in ensuring that questions related to the accuracy or integrity of any part of the work are appropriately investigated and resolved. Informed consent 
was not required because personal identifying information was not involved. We obtained the permission to access the SEER database with the ID number 19012-Nov2017 via Internet access method. Ethical approval was waived by the local ethics committee, as SEER data is publicly available, de-identified.

Open Access Statement: This is an Open Access article distributed in accordance with the Creative Commons Attribution-NonCommercial-NoDerivs 4.0 International License (CC BY-NC-ND 4.0), which permits the noncommercial replication and distribution of the article with the strict proviso that no changes or edits are made and the original work is properly cited (including links to both the formal publication through the relevant DOI and the license). See: https://creativecommons.org/licenses/by-nc-nd/4.0/.

\section{References}

1. Bray F, Ferlay J, Soerjomataram I, et al. Global cancer statistics 2018: GLOBOCAN estimates of incidence and mortality worldwide for 36 cancers in 185 countries. CA Cancer J Clin 2018;68:394-424.

2. Escudier B, Porta C, Schmidinger M, et al. Renal cell carcinoma: ESMO Clinical Practice Guidelines for diagnosis, treatment and follow-up. Ann Oncol 2019;30:706-20.

3. Shuch B, Bratslavsky G, Linehan WM, et al. Sarcomatoid renal cell carcinoma: a comprehensive review of the biology and current treatment strategies. Oncologist 2012;17:46-54.

4. Zhang L, Wu B, Zha Z, et al. The prognostic value and clinicopathological features of sarcomatoid differentiation in patients with renal cell carcinoma: a systematic review and meta-analysis. Cancer Manag Res 2018;10:1687-703.

5. Surveillance, Epidemiology, and End Results (SEER) Program (www.seer.cancer.gov) SEER*Stat Database: Incidence - SEER 18 Regs Research Data + Hurricane Katrina Impacted Louisiana Cases, Nov 2018 Sub (19752016 varying) - Linked To County Attributes - Total U.S., 1969-2017 Counties, National Cancer Institute, DCCPS, Surveillance Research Program, released April 2019, based on the November 2018 submission.

6. Ho DE, Imai K, King G, et al. MatchIt: Nonparametric Preprocessing for Parametric Casual Inference. Available online: http://www.jstatsoft.org/

7. Van Poppel H, Da Pozzo L, Albrecht W, et al. A prospective, randomised EORTC intergroup phase 3 study comparing the oncologic outcome of elective nephronsparing surgery and radical nephrectomy for low-stage renal cell carcinoma. Eur Urol 2011;59:543-52.

8. Shuch B, Bratslavsky G, Shih J, et al. Impact of pathological tumour characteristics in patients with sarcomatoid renal cell carcinoma. BJU Int 2012;109:1600-6.

9. Schieda N, Thornhill RE, Al-Subhi M, et al. Diagnosis of Sarcomatoid Renal Cell Carcinoma With CT: Evaluation by Qualitative Imaging Features and Texture Analysis. AJR Am J Roentgenol 2015;204:1013-23.

10. Merrill MM, Wood CG, Tannir NM, et al. Clinically nonmetastatic renal cell carcinoma with sarcomatoid dedifferentiation: Natural history and outcomes after surgical resection with curative intent. Urol Oncol 2015;33:166 e21-9.

11. Kim SP, Thompson RH, Boorjian SA, et al. Comparative effectiveness for survival and renal function of partial and radical nephrectomy for localized renal tumors: a systematic review and meta-analysis. J Urol 2012;188:51-7.

12. Shuch B, Hanley J, Lai J, et al. Overall survival advantage with partial nephrectomy: a bias of observational data? Cancer 2013;119:2981-9.

13. Scosyrev E, Messing EM, Sylvester R, et al. Exploratory Subgroup Analyses of Renal Function and Overall Survival in European Organization for Research and Treatment of Cancer randomized trial of Nephronsparing Surgery Versus Radical Nephrectomy. Eur Urol Focus 2017;3:599-605.

14. An JY, Ball MW, Gorin MA, et al. Partial vs Radical Nephrectomy for T1-T2 Renal Masses in the Elderly: Comparison of Complications, Renal Function, and Oncologic Outcomes. Urology 2017;100:151-7.

15. de Peralta-Venturina M, Moch H, Amin M, et al. Sarcomatoid differentiation in renal cell carcinoma: a study of 101 cases. Am J Surg Pathol 2001;25:275-84.

16. Capitanio U, Suardi N, Matloob R, et al. Extent of lymph node dissection at nephrectomy affects cancerspecific survival and metastatic progression in specific subcategories of patients with renal cell carcinoma (RCC). BJU Int 2014;114:210-5.

Cite this article as: Ji B, Li D, Fu S, Zhang Z, Yang T, Wu Y, Zuo Y, Xu Z, Yu N. Propensity-score matched comparison of partial versus radical nephrectomy for T1N0M0 sarcomatoid renal cell carcinoma. Transl Androl Urol 2020;9(2):250-257. doi: 10.21037/tau.2020.02.19 